$\xi=\mathbf{Z}$

\title{
Restricted three-body problem with stokes drag effect when less massive primary is an ellipsoid
}

\author{
M. Javed Idrisi ${ }^{1 *}$, Mamta Jain ${ }^{2}$ \\ ${ }^{1}$ Department of Mathematics, Al-Falah University, Faridabad (Haryana) - 121004, India \\ ${ }^{2}$ Department of Mathematics, ShriVenkateshwara University, U.P. - 244236, India \\ *Corresponding author E-mail:mjavedidrisi@gmail.com
}

\begin{abstract}
The present paper deals with the effect of Stokes drag force on the existence and stability of collinear and non-collinear libration points in circular restricted three-body problem when less massive primary is an ellipsoid. During the investigation, it is found that there exist five libration points $L_{i}(i=1,2 \ldots 5)$ out of which three are collinear and two are non-collinear. We observed that the Stokes drag force does not affect the collinear libration points while non-collinear libration points are affected by it and all the libration points either collinear or non-collinear are unstable in Lyapunov sense for the given range of dissipative constant $k$ and mass parameter $\mu$.
\end{abstract}

Keywords: Restricted Three Body Problem; Libration Points; Linear Stability; Stokes Drag.

\section{Introduction}

The restricted problem of three-body describes the motion of infinitesimal mass moving in the gravitational field of two massive primaries in the same plane or out of plane called two dimensional or three dimensional problem accordingly. The primaries are revolving around their center of mass either in circular or elliptical orbits under the influence of their mutual gravitational attraction. If the orbit of the primaries around their center of mass is elliptic, problem is said to be elliptic restricted three-body problem (ER3BP or ERTBP) and if the orbit of the primaries around their center of mass is circular, problem is said to be circular restricted three-body problem or restricted three-body problem, denoted by CR3BP or CRTBP or RTBP or R3BP.

The problem possesses five equilibrium points out of which three are collinear and two non-collinear. The collinear libration points are unstable while non-collinear are stable for the mass ratio $\mu \leq$ 0.038520896505 , Szebehely [2]. Some studies related to the equilibrium points in R3BP or ER3BP, taken into account the oblateness and triaxiality of the primaries, Coriolis and Centrifugal forces, Yarkovsky effect, variation of the masses of the primaries and the infinitesimal mass etc. are discussed by Danby [1]; Vidyakin [4]; Sharma [5]; Subbarao and Sharma [6]; Sharma and Subbarao [7]; Choudhary R. K. [8]; Cid R. et. al. [12]; El-Shaboury [16]; Bhatnagar et al. [19]; Selaru D. et.al. [21]; Markellos et al. [22]; Subbarao and Sharma [23]; Khanna and Bhatnagar [24, 25]; Roberts G.E. [28]; Oberti and Vienne [29]; Perdiou et. al. [30]; Sosnytskyi [31]; Ershkov [37]; Arredondo et.al. [38]; Idrisi and Taqvi [39]; Idrisi [40]; Idrisi and Amjad [42]; Idrisi [49]; Idrisi and Shalini [50].

The dust particles in the Solar System interact with the electromagnetic radiation field of the Sun as well as the solar gravitational field. According to Poynting (1903), when a dust particle is assumed to be spherical with a homogeneous structure, the solar radiation exerts a force on the particle in the direction of propagation of solar radiation, anti-parallel to solar gravity. Both radiation pressure and gravity acting on a homogeneous sphere approximately obey the inverse square law of distances from the center of the Sun. In Space science, the influence of the radiation is connected with the motion and the formation of concentrations of interplanetary and interstellar dust or grains in planetary and binary star systems. In 1937, Robertson gave a relativistic expression of the total radiation force on a particle by considering only linear terms in the ratio of the velocity of the particle over that of light as: $\boldsymbol{F}=F_{p} \boldsymbol{R} / R-F_{p}(\boldsymbol{v} . \boldsymbol{R}) \boldsymbol{R} / R-F_{p} v / c$

where $\mathbf{R}$ is the position vector of the particle $P$ with respect to a radiating source, $\boldsymbol{v}$ the corresponding velocity vector and $c$ the velocity of light, $F_{p}$ denotes the measure of the radiation pressure force. The first component in above equation expresses, the radiation pressure, while the remaining two components are the Doppler shift owing to the motion of the particle and the other component due to the absorption and subsequent re-emission of part of the incident radiation. These two components constitute the so called Poynting-Robertson effect. The P-R effect is important in the study of stability of the zodiacal cloud, asteroidal particles, dust rings around planets and orbital evolution of cometary meteor streams. The effect of P-R drag on the existence and stability of libration points is dicussed by many authors as Chernikov [3]; Murray [20]; Kushvah et.al. [32]; Lhotka and Celletti [46]; Mishra et. al. [47]; Pal et.al. [48].

The photo-gravitational restricted three-body problem arises from the classical problem if one or both primaries is an intense emitter of radiation, formulated by Radzievskii (1950). He has considered only the central forces of gravitation and radiation pressure on the particle of infinitesimal mass without considering the other two components of light pressure field and studied this problem for three specific bodies; the Sun, a planet and a dust particle. The radiation repulsive force $F_{p}$ exerted on a particle can be represented in terms of gravitational attraction $F_{\mathrm{g}}$ (Radzievskii, 1950) as $F_{p}$ $=F_{g}(1-q)$, where $q=1-F_{p} / F_{\mathrm{g}}$, a constant for a given particle, is a reduction factor expressed in terms of the particle radius $a$, density $\delta$ and radiation-pressure efficiency factor $x$ (in c.g.s. system) as: 


$$
q=1-\frac{5.6 \times 10^{-3}}{a \delta} x
$$

The assumption that ' $q$ ' is a constant implies that the fluctuations in the beam of solar radiation and the effect of planet's shadow are neglected. Typical values for diameter of IDP (Interplanetary Dust Particles) are in the range of $50-500 \mu \mathrm{m}$ and their densities range is $1-3 \mathrm{~g} / \mathrm{cm}^{3}$ with an average density of $2 \mathrm{~g} / \mathrm{cm}^{3}$. As the size of the particles increases, their density decreases (Grün et.al. [27]). Some of the notable research in PRTBP are carried by Chernikov [3]; Bhatnagar and Chawla [9]; Schuerman D.W. [10]; Kunitsyn and Tureshbaev [11]; Simmons et. al. [13]; Sharma [14]; Lukyanov [15]; Xuetang et.al. [18]; Ammar [33]; Douskos [34]; Singh and Leke [35]; Katour et.al. [41]; Singh and Amuda [45]; etc. In 2012, S. V. Ershkov studied the Yarkovsky effect in generalized photogravitational 3-body problem and proved the existence of maximally 256 different non-planar equilibrium points when second primary is non-oblate spheroid.

If a particle is moving in a gas, it experienced a force due to the collision of the particle with the molecules of the gas, called Stokes drag force. In planetary system, Stokes drag is used to describe the dissipative force acting on a particle moving around a star. The gas is assumed to be in circular motion around the barycenter of the system, its angular velocity is slightly smaller by a factor $\alpha$, than the Keplerian velocity at the same distance. The particle is assumed to be small or to move very slowly. In such a context, this effect can be described as dissipative force acting on the particle, modeled by a linear function of the relative velocity of the particle with respect to the gas. In synodic coordinates the components of the dissipative force are given by (Murray and Dermott [26]):

$\left(F_{x}, F_{y}\right)=-k\left(\dot{x}-y+\alpha \Omega_{y}, \dot{y}+x-\alpha \Omega_{x}\right)$

where $k \in[0,1)$ is the dissipative constant, depending on several physical parameters like the viscosity of gas, radius of the particle and mass of the particle; $\Omega=\Omega(r) \equiv r^{-3 / 2}$ is the Keplerian angular velocity at a distance $r=\left(x^{2}+y^{2}\right)^{1 / 2}$ from the origin of the synodic frame and $\alpha \in[0,1)$ is the ratio between the gas and Keplerian velocities (Murray [20])

The equations of motion of the infinitesimal mass in synodic coordinate system with Stokes drag effect are given by (Celletti et.al. [36]):

$$
\begin{aligned}
& \ddot{x}-2 \dot{y}=x-\frac{1-\mu}{r_{1}^{3}}(x-\mu)-\frac{\mu}{r_{2}^{3}}(x+1-\mu)-k\left(\dot{x}-y+\alpha \Omega_{y}\right) \\
& \ddot{y}+2 \dot{x}=y\left(1-\frac{1-\mu}{r_{1}^{3}}-\frac{\mu}{r_{2}^{3}}\right)-k\left(\dot{y}+x-\alpha \Omega_{x}\right)
\end{aligned}
$$

The restricted problem of three bodies with Stokes drag effect has been studied by Jain and Aggarwal ${ }^{[43]}$ considering primaries as a point mass and found that the there exist only two non-collinear libration points which are unstable due to Stokes drag effect Again, Jain and Aggarwal ${ }^{[44]}$ have discussed the existence and stability of non-collinear libration points under Stokes drag effect when smaller primary is an oblate body. They have shown that there exist two non-collinear libration points which are linearly unstable and collinear libration points do not exist because of Stokes drag effect.

In the present paper, we have studied the effect of Stokes drag on the existence and stability of libration points (collinear and noncollinear) considering a planar-circular and two dimensional restricted three body problem when less massive primary is an ellipsoid and mathematically we have proved that there exist three collinear libration points which are not affected by Stokes drag force, only non-collinear libration points are affected by Stokes drag force. The whole paper is divided into six sections. In section 2, Equations of motion of the infinitesimal mass under Stokes drag effect are obtained. In section 3, existence of collinear and noncollinear libration points have been shown. In section 4 , the stability of non-collinear libration points have been discussed. In sec- tion 5, an example to Sun-Earth system has been considered. In last section, conclusions are drawn.

\section{Equations of motion}

Let $m_{1}, m_{2}$ and $m_{3}$ be the masses of more massive primary, less massive primary and infinitesimal mass respectively and $a^{\prime}, b^{\prime}, c^{\prime}$ be the axes of the less massive primary $m_{2}$ such that $a^{\prime}>b^{\prime}>c^{\prime}$ with one of the axes as the axis of symmetry and its equatorial plane coinciding with the plane of motion. The primaries are revolving with angular velocity $n$ in circular orbits about their common centre of mass $O$ and $m_{3}$ is moving under the gravitational field of $m_{1}$ and $m_{2}$ in the same plane. The line joining $m_{1}$ and $m_{2}$ is taken as $X$-axis and ' $O$ ' their center of mass as origin and the line passing through $O$ and perpendicular to $O X$ and lying in the plane of motion of $m_{1}$ and $m_{2}$ is the $Y$-axis. We consider a synodic system of coordinates $O(x y z)$; initially coincident with the inertial system $O(X Y Z)$, rotating with the angular velocity $n$ about $Z$-axis (the $z$ axis is coincident with $Z$-axis) (Fig.1). The distances of $m_{3}$ from $m_{i}$ $(i=1,2)$ and $O$ are $\boldsymbol{r}_{\boldsymbol{i}}(i=1,2)$ and $\boldsymbol{r}$ respectively. Our aim is to find the equations of motion of $m_{3}$ using the terminology of Szebehely (1967) in the synodic co-ordinate system and dimensionless variables i.e. the distance between the primaries $m_{1}$ and $m_{2}$ is unity, the unit of time $t$ is such that the gravitational constant $G=1$ and the sum of the masses of the primaries is unity i.e. $m_{1}+m_{2}=1$.

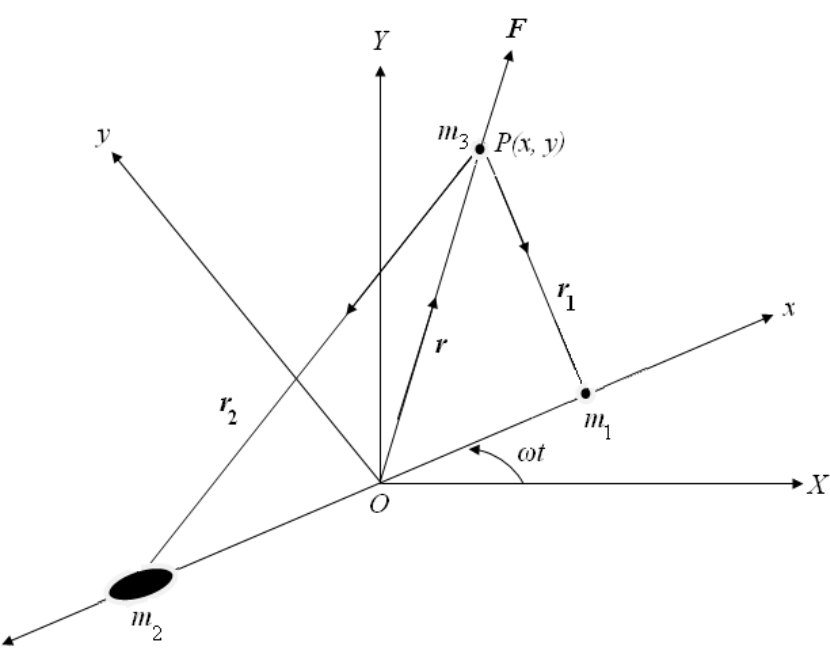

Fig. 1: Configuration of the Restricted Three Body Problem with Stokes Drag $\boldsymbol{F}$.

Using the terminology of circular restricted three-body problem, the equations of motion of the infinitesimal mass $m_{3}$ in the synodic coordinate system and dimensionless variables are given by

$$
\begin{aligned}
& \ddot{x}-2 n \dot{y}=\Omega_{x}+F_{x}, \\
& \ddot{y}+2 n \dot{x}=\Omega_{y}+F_{y} .
\end{aligned}
$$

where

$$
\begin{aligned}
\Omega= & \frac{n^{2}}{2}\left[(1-\mu) r_{1}^{2}+\mu r_{2}^{2}\right]+\frac{1-\mu}{r_{1}}+\frac{\mu}{r_{2}}+\frac{\mu}{2 r_{2}^{3}}\left(2 \sigma_{1}-\sigma_{2}\right)- \\
& \frac{3 \mu}{2 r_{2}^{5}}\left(\sigma_{1}-\sigma_{2}\right) y^{2}, \\
\Omega_{x}= & n^{2} x-(1-\mu) \frac{(x-\mu)}{r_{1}^{3}}-\mu \frac{(x+1-\mu)}{r_{2}^{3}} \times \\
& \left(1+\frac{3}{2 r_{2}^{2}}\left(2 \sigma_{1}-\sigma_{2}\right)-\frac{15}{2 r_{2}^{4}}\left(\sigma_{1}-\sigma_{2}\right) y^{2}\right),
\end{aligned}
$$


$\Omega_{y}=y\left[\begin{array}{l}n^{2}-\frac{(1-\mu)}{r_{1}^{3}}-\frac{\mu}{r_{2}^{3}} \times \\ \left(1+\frac{3}{2 r_{2}^{2}}\left(4 \sigma_{1}-3 \sigma_{2}\right)-\frac{15}{2 r_{2}^{4}}\left(\sigma_{1}-\sigma_{2}\right) y^{2}\right)\end{array}\right]$,

$n^{2}=1+\frac{3}{2}\left(2 \sigma_{1}-\sigma_{2}\right)$ is the mean motion of the primaries, (2)

$\sigma_{1}=\frac{a^{\prime 2}-c^{\prime 2}}{5}, \sigma_{2}=\frac{b^{\prime 2}-c^{\prime 2}}{5}$ are the oblateness factors,

$r_{1}^{2}=(x-\mu)^{2}+y^{2}$,

$r_{2}^{2}=(x+1-\mu)^{2}+y^{2}$,

$0<\mu=\frac{m_{2}}{m_{1}+m_{2}}<\frac{1}{2} \Rightarrow m_{1}=1-\mu ; \quad m_{2}=\mu$,

$S=S(r)=r^{-3 / 2}$ is the Keplerian angular velocity at distance $\boldsymbol{r}=x \boldsymbol{i}+$ $y \boldsymbol{j}$ from the origin of the synodic frame of reference and $\boldsymbol{\omega}=n \boldsymbol{k}$ is the angular velocity of the axes $O(x, y)$. Therefore, the components of Stokes drag along $x$ and $y$ axes are given by (Celletti et.al. [36]):

$F_{x}=-k\left(\dot{x}-y+\alpha S_{y}\right)$

$F_{y}=-k\left(\dot{y}+x-\alpha S_{x}\right)$

where $k$ is dissipative constant such that $0<k<1$ depends upon the various physical parameters as viscosity of the gas, radius of the particle, mass of the particle etc. and $0<\alpha<1$ is the ratio between the gas and Keplerian velocities.

If we put $k=0$ in Eqns. (1) and (2), the results are in conformity with those of Khanna and Bhatnagar [24].

Thus, the equations of motion (1) can be written as

$$
\begin{aligned}
& \ddot{x}-2 n \dot{y}=\Omega_{x}-k\left(\dot{x}-y+\alpha S_{y}\right), \\
& \ddot{y}+2 n \dot{x}=\Omega_{y}-k\left(\dot{y}+x-\alpha S_{x}\right) .
\end{aligned}
$$

The integral analogous to Jacobi integral is

$v^{2}=2(\Omega+F)-C$

where $v$ is the velocity of infinitesimal mass $m_{3}$ and $F$ is the Stokes drag whose components are given by (Murray and Dermott $\left.{ }^{[26]}\right)$.

\section{Libration points}

At the libration points, $\dot{x}=0, \dot{y}=0, \ddot{x}=0, \ddot{y}=0$, therefore from the Eqns. (5), we have

$$
\begin{aligned}
& n^{2} x-(1-\mu) \frac{(x-\mu)}{r_{1}^{3}}-\mu \frac{(x+1-\mu)}{r_{2}^{3}} \times \\
& \left(1+\frac{3}{2 r_{2}^{2}}\left(2 \sigma_{1}-\sigma_{2}\right)-\frac{15}{2 r_{2}^{4}}\left(\sigma_{1}-\sigma_{2}\right) y^{2}\right)+ \\
& k y\left(1+\frac{3}{2} \alpha\left(x^{2}+y^{2}\right)^{\frac{-7}{4}}\right)=0
\end{aligned}
$$

and

$$
\begin{aligned}
& y\left[n^{2}-\frac{(1-\mu)}{r_{1}^{3}}-\frac{\mu}{r_{2}^{3}}\left(\begin{array}{l}
\left.1+\frac{3}{2 r_{2}^{2}}\left(4 \sigma_{1}-3 \sigma_{2}\right)-\right) \\
\frac{15}{2 r_{2}^{4}}\left(\sigma_{1}-\sigma_{2}\right) y^{2}
\end{array}\right)\right]- \\
& k x\left(1+\frac{3}{2} \alpha\left(x^{2}+y^{2}\right)^{\frac{-7}{4}}\right)=0
\end{aligned}
$$

The collinear libration points are the solution of the Eqns. (6) and (7) for $y=0$ i.e

$$
\begin{aligned}
& n^{2} x-(1-\mu) \frac{(x-\mu)}{|x-\mu|^{3}}-\mu \frac{(x+1-\mu)}{|x+1-\mu|^{3}} \times \\
& \left(1+\frac{3}{2|x+1-\mu|^{2}}\left(2 \sigma_{1}-\sigma_{2}\right)\right)=0
\end{aligned}
$$

and

$$
k x\left(1+\frac{3}{2} \alpha\left(x^{2}\right)^{\frac{-7}{4}}\right)=0 .
$$

From Eqn. (9), $k \neq 0$ and $1+\frac{3}{2} \alpha\left(x^{2}\right)^{\frac{-7}{4}} \neq 0$, therefore $x=0$. It means the barycenter of the system itself a collinear librationpoint in case of Stoke's drag effect. Now, on substituting $x=0$ and $y=0$ in Eqn. (8) we have a fifth degree equation in $\mu$ i.e.

$$
4 \mu^{5}-14 \mu^{4}+\left(22+6 \sigma_{1}-3 \sigma_{2}\right) \mu^{3}-20 \mu^{2}+10 \mu-2=0
$$

Eqn. (10) has three real roots $\left(\mu_{i}, i=1,2,3\right)$ and all these three roots $\mu_{i}, \geq 1 / 2$ as shown in Fig. 2 but our assumption is $\mu<1 / 2$. Thus, barycenter can't be a collinear libration point in case of Stokes Drag. This is clear from the Eqn. (8), the collinear libration points are affected by only oblateness factors $\sigma_{1}$ and $\sigma_{2}$ not by the dissipative constant $k$ and the parameter $\alpha$ (ratio between the gas and Keplerian velocities). Since, this equation is solved by Khanna and Bhatnagar ${ }^{[24]}$ in detailed and they have proved that there are only three real solutions of the Eqn. (8) i.e. there exist only three collinear libration points $L_{i}(i=1,2,3)$ and the motion around these libration points is unbounded and consequently these libration points are linearly unstable for all values of $\mu, \sigma_{1}$ and $\sigma_{2}$. So, in next section we will skip the stability of the collinear libration points.

Thus, we conclude that, Stoke's drag does not affect collinear libration points, these points are affected by only oblateness factors $\sigma_{1}$ and $\sigma_{2}$ which are studied by Khanna and Bhatnagar [24].

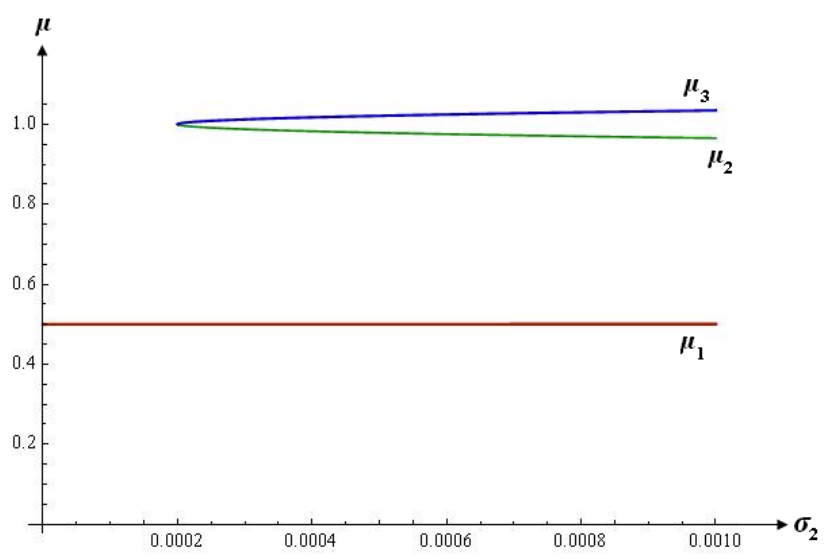

Fig. 2: $\sigma_{2}$ versus $\mu_{i}, i=1,2,3 ; \sigma_{1}=10^{-4}$

\subsection{Non-collinear libration points}

The non-collinear libration points are the solution of the Eqns. (6) and (7) for $y \neq 0$ i.e.

$$
\begin{aligned}
& n^{2} x-(1-\mu) \frac{(x-\mu)}{r_{1}^{3}}-\mu \frac{(x+1-\mu)}{r_{2}^{3}} \times \\
& \left(1+\frac{3}{2 r_{2}^{2}}\left(2 \sigma_{1}-\sigma_{2}\right)-\frac{15}{2 r_{2}^{4}}\left(\sigma_{1}-\sigma_{2}\right) y^{2}\right)+ \\
& k y\left(1+\frac{3}{2} \alpha\left(x^{2}+y^{2}\right)^{\frac{-7}{4}}\right)=0 \\
& \text { and }
\end{aligned}
$$

\subsection{Collinear libration points}


$y\left[n^{2}-\frac{(1-\mu)}{r_{1}^{3}}-\frac{\mu}{r_{2}^{3}}\left(\begin{array}{l}1+\frac{3}{2 r_{2}^{2}}\left(4 \sigma_{1}-3 \sigma_{2}\right)- \\ \frac{15}{2 r_{2}^{4}}\left(\sigma_{1}-\sigma_{2}\right) y^{2}\end{array}\right)\right]-$

$k x\left(1+\frac{3}{2} \alpha\left(x^{2}+y^{2}\right)^{\frac{-7}{4}}\right)=0$

On substituting $\sigma_{1}=0, \sigma_{2}=0$ and $k=0$, the solution of Eqns. (11) and (12) is $r_{1}=1, r_{2}=1$ and from Eqn. (2), $n=1$.

Now we assume that the solution of Eqns. (11) and (12) for $\sigma_{1} \neq 0$, $\sigma_{2} \neq 0$ and $k \neq 0$ as

$r_{1}=1+\xi_{1}, \quad r_{2}=1+\xi_{2}, \xi_{1}, \xi_{2}<<1$.

Substituting these values of $r_{1}$ and $r_{2}$ in the Eqns. (4) and (5), we get

$$
\begin{aligned}
& x=\mu-\frac{1}{2}+\xi_{2}-\xi_{1} \\
& y= \pm \frac{\sqrt{3}}{2}\left[1+\frac{2}{3}\left(\xi_{2}+\xi_{1}\right)\right]
\end{aligned}
$$

Now, substituting the values of $x, y$ from Eqns. (13), $r_{1}=1+\xi_{1}, r_{2}$ $=1+\xi_{2}$ in the Eqns. (11) and (12) and neglecting higher order terms, we obtain

$\xi_{1}=-\left[1-\frac{\mu}{2(1-\mu)}\right] \sigma_{1}+\frac{1}{2}\left[1-\frac{\mu}{(1-\mu)}\right] \sigma_{2}+\frac{(1+2 \mu)}{3 \sqrt{3}} k$ $\xi_{2}=-\frac{11}{8} \sigma_{1}+\frac{11}{8} \sigma_{2}-\frac{(2-\mu)}{3 \sqrt{3} \mu} k$.

Thus, the coordinates of the non-collinear libration points $L_{4,5}$ are

$$
\begin{gathered}
x=\mu-\frac{1}{2}-\frac{1}{2}\left[\frac{3}{4}+\frac{\mu}{1-\mu}\right] \sigma_{1}+\frac{1}{2}\left[\frac{7}{4}+\frac{\mu}{1-\mu}\right] \sigma_{2}-\frac{2}{3 \sqrt{3} \mu} k, \\
y= \pm \frac{\sqrt{3}}{2}\left[1+\frac{2}{3}\left\{\begin{array}{c}
\left(\frac{-19}{8}+\frac{\mu}{2(1-\mu)}\right) \sigma_{1}+ \\
\left(\frac{15}{8}-\frac{\mu}{2(1-\mu)}\right) \sigma_{2}-\frac{2(1-\mu)}{3 \sqrt{3} \mu} k
\end{array}\right\}\right]
\end{gathered}
$$

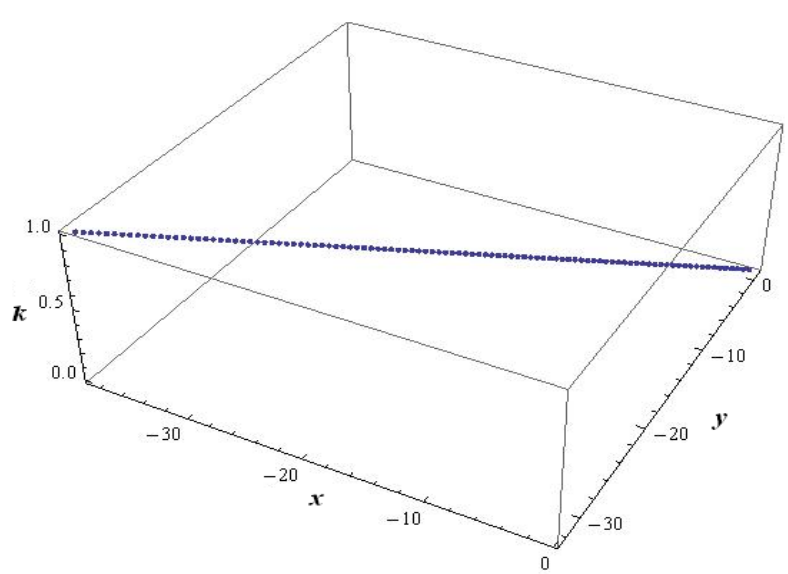

Fig. 3: $L_{4}(x, y)$ versus $k, \mu=0.01 ; \sigma_{1}=10^{-3} ; \sigma_{2}=10^{-4}$

As shown in the Fig. 3, the non-collinear libration points are affected by triaxiality as well as Stokes drag and these points move away from the center of mass as $k$ increases, also these points form scalene triangle with the primaries as $r_{1} \neq r_{2}$. If we put $k=0$ in Eqns. (14) and (15), the results are agreed with Khanna and Bhatnagar [24] and for $\sigma_{1}=0, \sigma_{2}=0$ and $k=0$, the results are in conformity with Szebehely [2].

\section{Stability of libration points}

The equations of the motion of the infinitesimal mass are

$$
\begin{aligned}
& \ddot{x}-2 n \dot{y}+k \dot{x}=\Omega_{x}+F_{x}, \\
& \ddot{y}+2 n \dot{x}+k \dot{y}=\Omega_{y}+F_{y} .
\end{aligned}
$$

To study the possible motion of the infinitesimal mass around the libration points let the coordinates of these points are $\left(x_{0}, y_{0}\right)$. If we give small displacement $(\zeta, \eta)$ to $\left(x_{0}, y_{0}\right)$ and considering only linear terms in $\zeta$ and $\eta$, the variation $\zeta$ and $\eta$ can be written as: $\zeta=x-x_{0}$ and $\eta=y-y_{0}$ and the equations of the motion become

$$
\begin{aligned}
\ddot{\varsigma}-2 n \dot{\eta}+k \dot{\varsigma} & =\Omega_{x}\left(x_{0}+\varsigma, y_{0}+\eta\right)+F_{x}\left(x_{0}+\varsigma, y_{0}+\eta\right) \\
& =\varsigma \Omega_{x x}^{o}+\eta \Omega_{x y}^{o}+\varsigma F_{x x}^{o}+\eta F_{x y}^{o}, \\
\ddot{\eta}+2 n \dot{\varsigma}+k \dot{\eta} & =\Omega_{y}\left(x_{0}+\varsigma, y_{0}+\eta\right)+F_{y}\left(x_{0}+\varsigma, y_{0}+\eta\right) \\
& =\varsigma \Omega_{y x}^{o}+\eta \Omega_{y y}^{o}+\varsigma F_{y x}^{o}+\eta F_{y y}^{o} .
\end{aligned}
$$

where $F_{x}=-k\left(-y+\alpha S_{y}\right), F_{y}=-k\left(x-\alpha S_{x}\right)$ and 'o' indicates that the partial derivatives are to be calculated at the libration points under consideration.

The characteristic equation of the Equations (16) is given by

$\lambda^{4}+a \lambda^{3}+b \lambda^{2}+c \lambda+d=0$

where

$a=2 k$,

$b=4 n^{2}+k^{2}-\stackrel{o}{\Omega}_{x x}-\stackrel{o}{\Omega}_{y y}-\stackrel{o}{F}_{x x}-\stackrel{o}{F}_{y y}$,

$c=2 n\left(\stackrel{o}{F}_{x y}-\stackrel{o}{F}_{y x}\right)-k\left(\stackrel{o}{\Omega}_{x x}+\stackrel{o}{\Omega}_{y y}+\stackrel{o}{F}_{x x}+\stackrel{o}{F}_{y y}\right)$,

$d=\stackrel{o}{\Omega}_{x x} \stackrel{o}{\Omega}_{y y}+\stackrel{o}{F}_{x x} \stackrel{o}{F}_{y y}+\stackrel{o}{F}_{x x} \stackrel{o}{\Omega}_{y y}+\stackrel{o}{F}_{y y} \stackrel{o}{\Omega}_{x x}-$

$\left(\stackrel{o}{\Omega_{x y}}\right)^{2}-\stackrel{o}{F_{y x}} \stackrel{o}{F_{x y}}-\stackrel{o}{\Omega_{x y}} \stackrel{o}{F_{x y}}-\stackrel{o}{\Omega_{x y}} \stackrel{o}{F_{y x}}$,

$\stackrel{o}{F}_{x x}=\frac{-21}{4} k \alpha x_{o} y_{o}\left(x_{o}^{2}+y_{o}^{2}\right)^{\frac{-11}{4}}$,

$\stackrel{o}{F}_{y y}=\frac{21}{4} k \alpha x_{o} y_{o}\left(x_{o}^{2}+y_{o}^{2}\right)^{\frac{-11}{4}}$

$\stackrel{o}{F}_{x y}=k\left(1+\frac{3}{2} \alpha\left(x_{o}^{2}+y_{o}{ }^{2}\right)^{\frac{-7}{4}}-\frac{21}{4} \alpha y_{o}{ }^{2}\left(x_{o}^{2}+y_{o}{ }^{2}\right)^{\frac{-11}{4}}\right)$,

$\stackrel{o}{F}_{y x}=-k\left(1+\frac{3}{2} \alpha\left(x_{o}{ }^{2}+y_{o}{ }^{2}\right)^{\frac{-7}{4}}+\frac{21}{4} \alpha x_{o}^{2}\left(x_{o}{ }^{2}+y_{o}{ }^{2}\right)^{\frac{-11}{4}}\right)$,

$\stackrel{o}{\Omega}_{x x}=n^{2}-\frac{1-\mu}{r_{1}^{\prime 3}}\left(1-\frac{3\left(x_{o}-\mu\right)^{2}}{r_{1}^{\prime 2}}\right)-\frac{\mu}{r_{2}^{\prime 3}}\left(1-\frac{3\left(x_{o}+1-\mu\right)^{2}}{r_{2}^{\prime 2}}\right)-$

$\frac{3 \mu}{2 r_{2}^{\prime 5}}\left(2 \sigma_{1}-\sigma_{2}\right)\left(1-\frac{5\left(x_{o}+1-\mu\right)^{2}}{r_{2}^{\prime 2}}\right)+$

$\frac{15 \mu}{2 r_{2}^{\prime 7}} y_{0}^{2}\left(\sigma_{1}-\sigma_{2}\right)\left(1-\frac{7\left(x_{o}+1-\mu\right)^{2}}{r_{2}^{\prime 2}}\right)$, 


$$
\begin{aligned}
& \stackrel{o}{\Omega_{x y}}=\frac{3(1-\mu)\left(x_{o}-\mu\right) y_{o}}{r_{1}^{\prime 5}}+\frac{3 \mu\left(x_{o}+1-\mu\right) y_{o}}{r_{2}^{\prime 5}}+ \\
& \frac{15 \mu\left(x_{o}+1-\mu\right) y_{o}}{2 r_{2}^{\prime 7}}\left(2 \sigma_{1}-\sigma_{2}\right)+ \\
& \frac{15 \mu\left(x_{o}+1-\mu\right) y_{o}}{r_{2}^{\prime 7}}\left(\sigma_{1}-\sigma_{2}\right)- \\
& \frac{105 \mu\left(x_{o}+1-\mu\right) y_{o}{ }^{3}}{r_{2}^{\prime 9}}\left(\sigma_{1}-\sigma_{2}\right) \text {, } \\
& \stackrel{o}{\Omega_{y y}}=n^{2}-\frac{(1-\mu)}{r_{1}^{\prime 3}}-\frac{\mu}{r_{2}^{\prime 3}}\left(\begin{array}{l}
1+\frac{3}{2 r_{2}^{\prime 2}}\left(4 \sigma_{1}-3 \sigma_{2}\right)- \\
\frac{15}{2 r_{2}^{\prime 4}}\left(\sigma_{1}-\sigma_{2}\right) y_{o}{ }^{2}
\end{array}\right)+ \\
& y_{o}\left[\begin{array}{l}
\frac{3(1-\mu) y_{0}}{r_{1}^{\prime 5}}+\frac{3 \mu y_{0}}{r_{2}^{\prime 5}}-\frac{15 \mu y_{o}}{2 r_{2}^{\prime 7}}\left(4 \sigma_{1}-3 \sigma_{2}\right)+ \\
\frac{15 \mu y_{o}}{r_{2}^{\prime 7}}\left(\sigma_{1}-\sigma_{2}\right)-\frac{105 \mu y_{o}^{3}}{2 r_{2}^{\prime 9}}\left(\sigma_{1}-\sigma_{2}\right)
\end{array}\right],
\end{aligned}
$$$$
\text { 4.1 Stability of Non-Collinear Libration Point } \boldsymbol{L}_{\mathbf{4}}
$$

At the non-collinear libration points $L_{4}$

$$
\begin{aligned}
x_{o}= & \mu-\frac{1}{2}-\frac{1}{2}\left[\frac{3}{4}+\frac{\mu}{1-\mu}\right] \sigma_{1}+ \\
& \frac{1}{2}\left[\frac{7}{4}+\frac{\mu}{1-\mu}\right] \sigma_{2}-\frac{2(1-\mu)}{3 \sqrt{3} \mu} k,
\end{aligned}
$$

and

$$
y_{o}= \pm \frac{\sqrt{3}}{2}\left[1+\frac{2}{3}\left\{\begin{array}{l}
\left(\frac{-19}{8}+\frac{\mu}{2(1-\mu)}\right) \sigma_{1}+ \\
\left(\frac{15}{8}-\frac{\mu}{2(1-\mu)}\right) \sigma_{2}-\frac{2}{3 \sqrt{3} \mu} k
\end{array}\right\}\right]
$$

As shown in the Fig. 4 , in the non-shaded region $\Delta>0, P>0$ while in light-shaded region $\Delta<0, P>0$ and in dark-shaded region $\Delta<0, P<0$ in the interval $0<\mu<1 / 2,0<k<1$. Thus, the characteristic Eqn. (17) has complex conjugate roots in nonshaded region, in light-shaded region two roots are real while two roots are complex, in dark-shaded region all roots are real and distinct. Hence, the non-collinear libration point $L_{4}$ is unstable in 0 $<\mu<1 / 2,0<k<1$. Similarly, we can show that $\mathrm{L}_{5}$ is also unstable.

$$
\begin{aligned}
& r_{1}^{\prime 2}=\left(x_{o}-\mu\right)^{2}+y_{o}^{2}, \\
& r_{2}^{\prime 2}=\left(x_{o}+1-\mu\right)^{2}+y_{o}^{2} .
\end{aligned}
$$

The Eqn. (17) is a quartic equation in $\lambda$ and its discriminant is given by (https://en.wikipedia.org/wiki/Quartic_function)

$$
\begin{gathered}
\Delta=256 d^{3}-192 a c d^{2}-128 b^{2} d^{2}+144 b c^{2} d- \\
27 c^{4}+144 a^{2} b d^{2}-6 a^{2} c^{2} d-80 a b^{2} c d+ \\
18 a b c^{3}+16 b^{4} d-4 b^{3} c^{2}-27 a^{4} d^{2}+ \\
18 a^{3} b c d-4 a^{3} c^{3}-4 a^{2} b^{3} d+a^{2} b^{2} c^{2},
\end{gathered}
$$

The nature of roots depends upon $\Delta$, given as:

- If $\Delta<0$, two roots are real and two complex conjugate roots

- If $\Delta>0$, all roots are real or complex

$\circ \quad \mathrm{P}<0$ and $\mathrm{D}<0$, all roots are real and distinct

- $\mathrm{P}>0$ or $\mathrm{D}>0$, there are two pairs of complex conjugate roots

- If $\Delta=0$ and

$\circ \quad \mathrm{P}<0, \mathrm{D}<0$ and $\Delta_{\mathrm{o}} \neq 0$, there is a real double root and two simple real roots

$\circ \quad \mathrm{P}>0$ or $\mathrm{D}>0$, there is a real double root and two complex conjugate roots

- $\Delta_{\mathrm{o}}=0$, there is a triple real root and a simple real root

- If $\mathrm{D}=0$ and

- $\quad \mathrm{P}<0$, there are two real double roots

- $\quad \mathrm{P}>0$ and $\mathrm{Q}=0$, there are two complex conjugate double roots

where

○ $\Delta_{\mathrm{o}}=0$, all four roots are equal to $-a / 4$

$$
\begin{aligned}
& P=8 b-3 a^{2}, \\
& Q=a^{3}+8 c-4 a b, \\
& \Delta_{o}=b^{2}-3 a c+12 d, \\
& D=64 d-16 b^{2}+16 a^{2} b-16 a c-3 a^{4} .
\end{aligned}
$$

The libration point $\left(x_{0}, y_{0}\right)$ is said to be stable if all four roots $\lambda_{i}(i$ $=1,2,3,4$ ) have negative real values or pure imaginary.

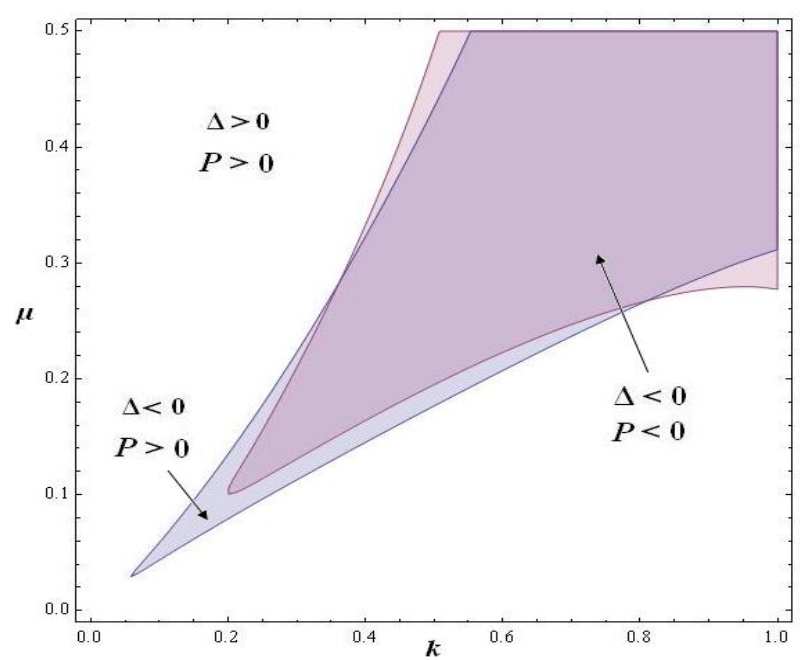

Fig. 4: $\Delta-P$ region with respect to $\mu$ and $k ; \alpha=0.05, \sigma_{1}=10^{-3}, \sigma_{2}=10^{-4}$

\section{Application to sun-earth system}

Let us consider an example of the Sun-Earth system in planar restricted three-body problem considering the smaller primary $m_{2}$ i.e. ellipsoid as Earth and the bigger one $m_{1}$ as Sun. From the astrophysical data (Lang [17]) we have, mass of the Sun $\left(m_{1}\right)=$ $1.9891 \times 10^{30} \mathrm{~kg}$; mass of the Earth $\left(m_{2}\right)=5.9742 \times 10^{24} \mathrm{~kg}$; axes of the Earth: $a=6378.140 \mathrm{~km}, b=6368 \mathrm{~km}$ and $c=6356.755 \mathrm{~km}$ Mean distance of Earth from the Sun $=1.49598 \times 10^{8} \mathrm{~km}$. In dimensionless system: $\mu=0.00000300346 ; a_{1}=0.0000426352 ; b_{1}=$ $0.0000425675 ; c_{1}=0.0000424923$. Therefore, from the Eqn. (2), the mean-motion of the primaries is $n=1.0000000000026898$. The location of non-collinear libration point $L_{4}$ from the Eqns. (14) and (15) is plotted in Fig. 5. The abscissa and ordinate both are decreasing functions with respect to $k$ for the non-collinear libration point $L_{4}$ in Sun-Earth System. 


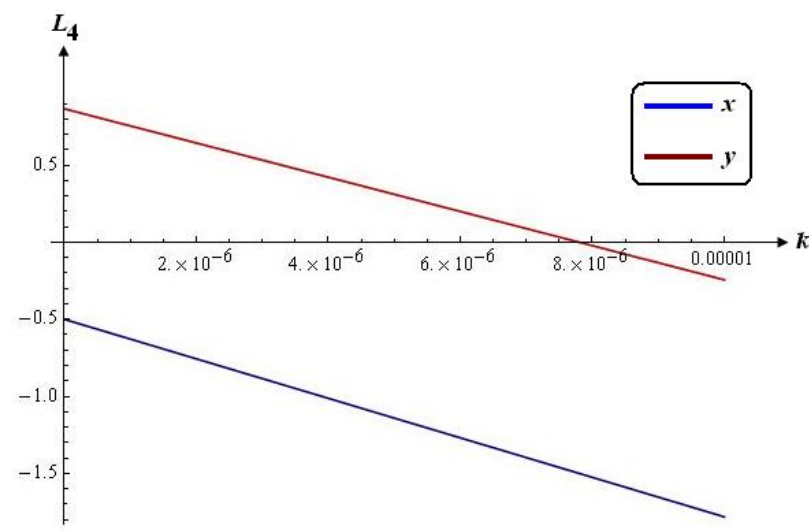

Fig. 5: $L_{4}$ in Sun-Earth System

Now, to discuss the stability of the collinear libration point $L_{4}$ we have plotted the $\Delta$ and $P$ curves for different values of $k$. As shown in Fig. $6, \Delta>0$ and $P>0$ which implies that the characteristic Eqn. (17) has complex conjugate roots in the interval $0<k<$ 1. Thus, the non-collinear libration point $L_{4}$ in Sun-Earth System is unstable. Similarly, $L_{5}$ is also unstable in $0<k<1$.

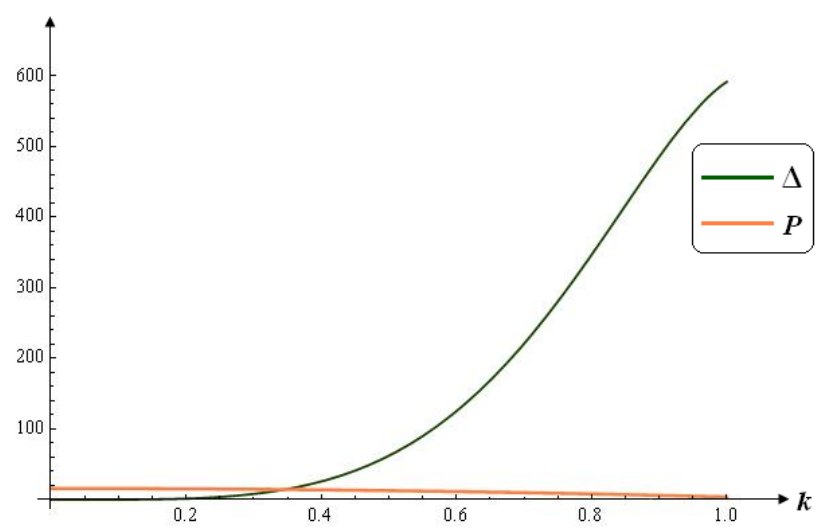

Fig. 6: $k$ versus $\Delta$ and $P ; \alpha=0.05$

\section{Conclusion}

In the present paper, we have considered a planar circular restricted three body problem when less massive primary is an ellipsoid and studied the effect of Stokes drag on the infinitesimal mass $m_{3}$. This is found that, there exist five libration points $L_{i}(i=1,2,3,4,5)$ out of which three are collinear and affected by only oblateness factors of ellipsoid i.e. $\sigma_{1}$ and $\sigma_{2}$. The non-collinear libration points $L_{4}$ and $L_{5}$ are affected by oblateness as well as Stokes drag. The abscissa and ordinate of non-collinear libration points $L_{4,5}$ are decreasing functions with respect to $k$. Further, all the libration points either collinear or non-collinear all are unstable in Lyapunov sense. Finally we considered an application to Sun-Earth system and found that the non-collinear libration points exists and unstable for $0<k<1$. In our case, when $\sigma_{1}=0, \sigma_{2}=0$ and $k=0$ the results are in agreement with the classical case of restricted three-body problem (Szebehely [2]) and if $\sigma_{1} \neq 0, \sigma_{2} \neq 0, \sigma_{1} \neq \sigma_{2}$ and $k=0$ the results are in conformity with Khanna and Bhatnagar [24].

\section{References}

[1] Danby, J.M.A.: Stability of the triangular points in the elliptic restricted problem of three bodies. The Astronomical Journal, 69 (2), 165 - 172 (1964).http://dx.doi.org/10.1086/109254.

[2] Szebehely, V.: Theory of orbits, The Restricted Problem of three bodies. Academic Press, New York and London (1967).

[3] Chernikov, Y.A.: The photogravitational restricted three body problem. Soviet Astronomy-AJ, 14, 176-181 (1970).
[4] Vidyakin, V.V.: Stability of one particular solution for the motion of three homogeneous spheroids.Soviet Astronomy, 18, 116 (1974).

[5] Sharma, R.K.: Perturbations of Lagrangian points in the restricted three-body problem. Indian Journal of Pure and Applied Mathematics, 6, 1099-1102 (1975).

[6] Subbarao, P.V., Sharma, R.K.: A note on the Stability of the triangular points of equilibrium in the restricted three body problem. Astronomy and Astrophysics, 43, 381-383 (1975).

[7] Sharma, R.K., Subbarao P.V.: Stationary solutions and their characteristic exponents in the restricted three-body problem when the more massive primary is an oblate spheroid. Celestial Mechanics and Dynamical Astronomy, 13, 137-149 (1976). http://dx.doi.org/10.1007/BF01232721.

[8] Choudhary R.K.: Libration points in the generalized elliptic restricted three body problem. Celestial Mechanics, 16, $411-419$ (1977). http://dx.doi.org/10.1007/BF01229285.

[9] Bhatnagar, K.B., Chawla, J.M.: A study of Lagrangian points in the photogravitational restricted three-body problem. Indian J. Pure Appl. Math. 10(11), 1443-1451 (1979).

[10] Schuerman, D.W.: The restricted three body problem including radiation pressure. The Astrophysical Journal, 238, 337-342 (1980). http://dx.doi.org/10.1086/157989.

[11] Kunitsyn, A.L., Tureshbaev, A.T.: On the collinear libration points in the photogravitational three-body problem. Celest. Mech. 35(2), 105-112 (1985). http://dx.doi.org/10.1007/BF01227664.

[12] Cid, R., Ferrer, S., Caballero, J.A.: Asymptotic solutions of the restricted problem near equilateral Lagrangian points. Celestial Mechanics and Dynamical Astronomy, 35, 189-200 (1985). http://dx.doi.org/10.1007/BF01227668.

[13] Simmons, J.F.L., McDonald, A.J.C., Brown, J.C.: The restricted 3body problem with radiation pressure. Celestial Mechanics and Dy-

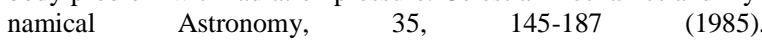
http://dx.doi.org/10.1007/BF01227667.

[14] Sharma, R.K.: The linear stability of libration points of the photogravitational restricted three-body problem when the smaller primary is an oblate spheroid. Astrophys. Space Sci. 135(2), 271-281 (1987). http://dx.doi.org/10.1007/BF00641562.

[15] Lukyanov, L.G.: On the family of the libration points in the restricted photogravitational three-body problem. Astron. Zh. 65, 422-432 (1988).

[16] El-Shaboury, S.M.: Equilibrium solutions of the restricted problem of $2+2$ axisymmetric rigid bodies. Celestial Mechanics and Dynamical Astronomy, 50, 199-208 (1991). http://dx.doi.org/10.1007/BF00048764.

[17] Lang, K. R.: Astrophysical Data: Planets and Stars. SpringerVerlag, New York (1992). http://dx.doi.org/10.1007/978-1-46840640-5.

[18] Xuetang, Z.H.E.N.G., Lizhong, Y.U.: Photogravitationally restricted three-body problem and coplanar libration point. Chinese Phys. Lett., 10 (1), 61 - 64 (1993). http://dx.doi.org/10.1088/0256307X/10/1/017.

[19] Bhatnagar, K.B., Gupta, U., Bharadwaj, R.: Effect of perturbed potentials on the non-linear stability of Libration point $\mathrm{L}_{4}$ in the restricted problem. Celestial Mechanics and Dynamical Astronomy, 59, 45-374 (1994). http://dx.doi.org/10.1007/BF00692102.

[20] Murray, C.D.: Dynamical effects of drag in the circular restricted three body problems: 1.Location and stability of the Lagrangian equilibrium points. Icarus 112, 465-184 (1994) http://dx.doi.org/10.1006/icar.1994.1198.

[21] Selaru, D., Cucu-Dumitrescu, C.: Infinitesimal orbits around Lagrange points in the elliptic, restricted three-body problem. Celestial Mechanics and Dynamical Astronomy, 61 (4), 333 - 346 (1995). http://dx.doi.org/10.1007/BF00049514.

[22] Markellos, V.V., Papadakis, K.E., Perdios, E.A.: Non-linear stability zones around triangular equilibria in the plane circular restricted three-body problem with oblateness. Astrophysics and Space Science, 245, 157-164 (1996). http://dx.doi.org/10.1007/BF00637811.

[23] Subbarao, P.V., Sharma, R.K.: Effect of oblateness on the nonlinear stability of $\mathrm{L}_{4}$ in the restricted three-body problem. Celestial Mechanics and Dynamical Astronomy, 65, 291-312 (1997).

[24] Khanna, M., Bhatnagar, K.B.: Existence and stability of libration points in restricted three body problem when the smaller primary is a triaxial rigid body. Indian Journal of Pure and Applied Mathematics, 29 (10), 1011-1023 (1998).

[25] Khanna, M., Bhatnagar, K.B.: Existence and stability of Libration points in the restricted three body problem when the smaller primary is a triaxial rigid body and the bigger one an oblate spheroid. Indian Journal of Pure and Applied Mathematics, 30 (7), 721-733 (1999). 
[26] Murray, C.D., Dermott, S.F.: Solar System Dynamics. Cambridge University Press, Cambridge (1999).

[27] Grün, E., Gustafson, B.A.S., Dermott, S., Fechtig, H.: Interplanetary Dust. Springer, Berlin (2001).http://dx.doi.org/10.1007/978-3642-56428-4.

[28] Roberts, G.E.: Linear Stability of the Elliptic Lagrangian Triangle Solutions in the three-body problem. Journal of Differential Equa$\begin{array}{llllll}\text { tions, } & 182 & \text { (1), } & 191 & - & 218\end{array}$ http://dx.doi.org/10.1006/jdeq.2001.4089.

[29] Oberti, P.,Vienne, A.: An upgraded theory for Helene, Telesto and Calypso. Astronomy and Astrophysics, 397, 353-359 (2003).http://dx.doi.org/10.1051/0004-6361:20021518.

[30] Perdiou, A.E., Markellos, V.V., Douskos, C.N.: The Hill problem with oblate secondary: Numerical Exploration. Earth, Moon and Planets, 97, 127-145 (2005). http://dx.doi.org/10.1007/s11038-0069065-y.

[31] Sosnytskyi, S.P.: On the Lagrange stability of motion in the threebody problem. Ukrainian Mathematical Journal, 57 (8), 1341-1349 (2005). http://dx.doi.org/10.1007/s11253-005-0266-8.

[32] Kushvah, B.S., Sharma, J.P., Ishwar, B.: Nonlinear Stability in the Generalized Photogravitational Restricted Three Body Problem with Poynting-Robertson Drag. Astrophysics and Space Science, 312, 279-293 (2007). http://dx.doi.org/10.1007/s10509-007-9688-0.

[33] Ammar, M.K.: The effect of solar radiation pressure on the Lagrangian points in the elliptic restricted three-body problem. Astrophysics and Space Science, 313 (4), 393 - 408 (2008). http://dx.doi.org/10.1007/s10509-007-9709-z.

[34] Douskos, C.N.: Collinear equilibrium points of Hill's problem with radiation and oblateness and their fractal basins of attraction. Astrophysics and Space Science, 326, 263-271 (2010) http://dx.doi.org/10.1007/s10509-009-0213-5.

[35] Singh, Jagdish, Leke, O.: Stability of the photogravitational re stricted three body problem with variable masses. Astrophysics and Space Science, 326, 305-314 (2010). http://dx.doi.org/10.1007/s10509-009-0253-x.

[36] Celletti, A., Stefanelli, L., Lega, E., Froeschlé, C.: Some Results on the Global Dynamics of the Regularized Restricted Three-Body Problem with Dissipation. Celestial Mechanics and Dynamical Astronomy, 109, 265-284 (2011). http://dx.doi.org/10.1007/s10569010-9326-y.

[37] Ershkov, Sergey V.: The Yarkovsky effect in generalized photogravitational 3-body problem. Planetary and Space Science, 70 (1), 221-223 (2012). http://dx.doi.org/10.1016/i.pss.2012.09.002.

[38] John, A. Arredondo, Jianguang, Guo, Cristina, Stoica, Claudia Tamayo: On the restricted three body problem with oblate primaries. Astrophysics and Space Science, 341, 315-322 (2012). http://dx.doi.org/10.1007/s10509-012-1085-7.

[39] Idrisi, M. Javed, Taqvi, Z.A.: Restricted three-body problem when one of the primaries is an ellipsoid. Astrophysics and Space Science, 348, 41-56 (2013). http://dx.doi.org/10.1007/s10509-0131534-y.

[40] Idrisi, M. Javed: Existence and stability of the libration points in CR3BP when the smaller primary is an oblate spheroid. Astrophysics and Space Science, 354, 311-325 (2014). http://dx.doi.org/10.1007/s10509-014-2031-7.

[41] Katour, D.A., Abd El-Salam, F.A., Shaker, M.O.: Relativistic restricted three body problem with oblateness and photo-gravitational corrections to triangular equilibrium points. Astrophysics and Space Science, 351, 143 - 149 (2014). http://dx.doi.org/10.1007/s10509014-1826-x.

[42] Idrisi, M. Javed, Amjad, Muhammad: Effect of elliptic angle $\varphi$ on the existence and stability of libration points in restricted threebody problem in earth-moon system considering earth as an ellipsoid. International Journal of Advanced Astronomy, 3 (2),87-96 (2015). http://dx.doi.org/10.14419/ijaa.v3i2.5313.

[43] Jain, Mamta, Aggarwal, Rajiv: Restricted three body problem with Stokes Drag effect. International Journal of Astronomy and Astrophysics, 5, 95-105 (2015). http://dx.doi.org/10.4236/ijaa.2015.52013.

[44] Jain, Mamta, Aggarwal, Rajiv: A study of non-collinear libration points in restricted three body problem with stokes drag effect when smaller primary is an oblate spheroid. Astrophysics and Space Science, 358: 51 (2015). http://dx.doi.org/10.1007/s10509-015-2457-6.

[45] Singh, J., Amuda, T.O.: Out-of-plane equilibrium points in the photogravitational CR3BP with oblateness and P-R drag. Journal of Astrophysics and Astronomy, 36, 291-305 (2015). http://dx.doi.org/10.1007/s12036-015-9336-y.

[46] Lhotka, C., Celletti, A.: The effect of Poynting Robertson drag on the triangular Lagrangian points. Astrophysics and Space Science, 250, 249-261 (2015).http://dx.doi.org/10.1016/j.icarus.2014.11.039.
[47] Mishra, Vivek Kumar, Ishwar, Bhola: Normalisation of Hamiltonian in photogravitational elliptic restricted three body problem with Poynting-Robertson drag. International Journal of Advanced Astroomy, 3, 42-45 (2015). http://dx.doi.org/10.14419/ijaa.v3i1.4534.

[48] Pal, Ashok Kumar, Kushvah, Badam Singh: Geometry of halo and lissajous orbits in the circular restricted three body problem with drag forces.MNRAS446, 959-972 (2015).

[49] Idrisi, M. Javed: A general solution to non-collinear equilibria in terms of largest root $(\kappa)$ of confocal oblate spheroid. International Journal of Advanced Astronomy, 4 (1), 1 - 4 (2016). http://dx.doi.org/10.14419/ijaa.v4i1.5587.

[50] Idrisi, M. Javed, Shalini, Kumari: Non-collinear libration points in CR3BP when less massive primary is an heterogeneous oblate body with N-layers. International Journal of Advanced Astronomy, 4 (1), 39 - 42 (2016). http://dx.doi.org/10.14419/ijaa.v4i1.5928. 